\title{
Applications of laser Raman and FT-IR techniques to leprology, neurology, bacteriology and traumatology
}

\author{
S MISHRA, RAVINDRA KUMAR, S K ARYAL, S KUMAR, \\ RAJESH KUMAR and M M BAJAJ* \\ Medical Physics Research Laboratory, Department of Physics and Astrophysics, University \\ of Delhi, Delhi 110007 , India
}

\begin{abstract}
The application of Fourier transform infrared spectroscopy and laser Raman spectroscopy for obtaining structural information about the subtle changes occurring at the molecular level following leprosy, pulmonary tuberculosis, infected burns, epilepsy and Duchenne muscular dystrophy (DMD) are reported in this paper. It is shown that IR spectra exhibit specific signals for each disease. Results of the observed bands of hemoproteins are compared with those of normals. Raman spectra of $\operatorname{IgG}$ reveal that alpha-helical conformations are introduced partially in case of the proliferation of Mycobacterium leprae. These results are supported by our IR studies. Typical signals of perturbed stretching region are seen in the pulmonary tuberculosis cases. Disappearance of a few normal bands and strong peaks in the hydrocarbon region characterizes epileptic disorder. Perturbations in the Raman spectra of erythrocyte ghosts are identified following gram-negative Pseudomonas aeruginosa infection. Investigations of pathological changes following the onset of body disorders at the molecular level are reported using IR and Raman spectroscopic techniques.
\end{abstract}

Keywords. Raman/FT-IR spectroscopy; Duchenne muscular dystrophy; neurology; epilepsy; leprosy; tuberculosis; bacteriology; traumatology.

\section{Introduction}

Alterations in the lipid and protein components have been found to be responsible for the change in the Raman spectra of erythrocyte membranes upon varying $\mathrm{pH}$ and temperature, whereas specific changes in the $\mathrm{C}-\mathrm{H}$ stretching region are reported to be related to the alterations in the integral protein environment. Earlier Stewart et al (1960) employed IR spectroscopy to determine the ethanol and carbon tetrachloride in blood, whereas Freeman (1966) used it to determine strains of tuberculosis bacilli.

Till now most of the information concerning the conformation of immunoglobulin (IgG) has come from X-ray, circular dichroism (CD) and infrared spectroscopy. It has been shown by several workers (Abaturov et al 1969; Litman et al 1973; Amzel and Poljak 1979) that the immunoglobulin contains the beta-structure and irregular conformation but not the alpha-helical conformation. Abaturov et al (1969) have reported the observed frequencies of the four amide bands of $\mathrm{IgG}$, its peptide chains and proteolytic fragments. The purpose of this work is to show that laser Raman

*For correspondence 
and FT-IR spectroscopies can be used to understand and characterize the conformational changes occurring at the molecular and electronic level, following perturbations caused by proliferating agents like $M$ ycobacterium leprae, Mycobacterium tuberculosis and Pseudomonas aeruginosa infections (Verma and Wallach 1975; Harvey et al 1976; Nikkelson et al 1978; Wallach et al 1979; Somani 1984). DMD is an X-linked, recessive neuromuscular disorder with an incidence of 1 in 3500 (Kumar and Bajaj 1990). Sato et al (1978) found marked distinction between the membranes from normal and DMD erythrocytes in terms of temperature dependence, $\mathrm{pH}$ dependence and accessibility to reduction by ascorbate. Wilkerson et al (1978) suggested that DMD membranes are abnormal in terms of membrane fluidity or lipiprotein interactions. We have carried out Raman studies comparing plasma membranes from normal and DMD affected persons in the region $800-1200 \mathrm{~cm}^{-1}$.

\section{Materials and methods}

The blood samples from tubercular, leprous, burn and neurological cases were collected from different hospitals. Red cells were dehydrated over phosphorus pentoxide. The samples were prepared by the methods given by Fairbanks et al (1971) and Bajaj and Khandelwal (1985). The separation of IgG was carried out by the ion exchange chromatographic technique (Wier 1980).

Immunoglobulin samples were lyophilized and the $\mathrm{IgG}$ so obtained was further tested for its purity with the help of antisera (Hoechst) using the single radial immunodiffusion technique (Oucherlony 1962). After the separation, $10 \mathrm{mg}$, of the sample was dissolved in $\mathrm{D}_{2} \mathrm{O}$ and transferred to the sample cell container.

The infrared spectra of prepared samples were recorded with single beam Fourier Transform-Infrared Spectrophotometer (Perkin-Elmer model-1710). Raman spectra of heme proteins, immunoglobulin and erythrocyte ghosts were recorded in the $500-3000 \mathrm{~cm}^{-1}$ region with a laser Raman Spectrophotometer (Model NR-1000) consisting of photomultiplier unit ( $R-464)$ including Argon ion laser at $514.5 \mathrm{~nm}$ having scanning speed of $20 \mathrm{~cm}^{-1} \mathrm{~min}^{-1}$ (figures 1 and 2).

\section{Results and discussion}

Results obtained from the FTIR studies of normal and pathological samples demarcate specific regions for a particular type of disorder. In pulmonary tuberculosis (PTB) cases, strong signals at 3686,3679 and $3608 \mathrm{~cm}^{-1}$ are observed in the lattice water region arising due to $\mathrm{O}-\mathrm{H}$ bond whereas in the lepromatus leprosy (LL) cases, peaks around 3461,3445 and $3442 \mathrm{~cm}^{-1}$ are found in the same region. Amide B bands are found in PTB cases at 3083, 3082 and $3064 \mathrm{~cm}^{-1}$ whereas these bands are absent in LL cases. A strong peak at $2930 \mathrm{~cm}^{-1}$ appears with a shoulder at $2915 \mathrm{~cm}^{-1}$ in the (PTB) cases (figure 1). In the normal group the $2871 \mathrm{~cm}^{-1}$ band is shifted in PTB cases towards lower energy and in infected burn cases, towards the higher side at $2860 \mathrm{~cm}^{-1}$. Typical IR signals are observed in LL cases at 2068 and $2067 \mathrm{~cm}^{-1}$.

Strong peaks occur in amide I, amide II region at 1640 and $1560 \mathrm{~cm}^{-1}$ for heme proteins of burn patients whereas in normal samples signals are observed around $1596 \mathrm{~cm}^{-1}$. In the amide IV to VI stretching region, a peculiar peak is observed at 


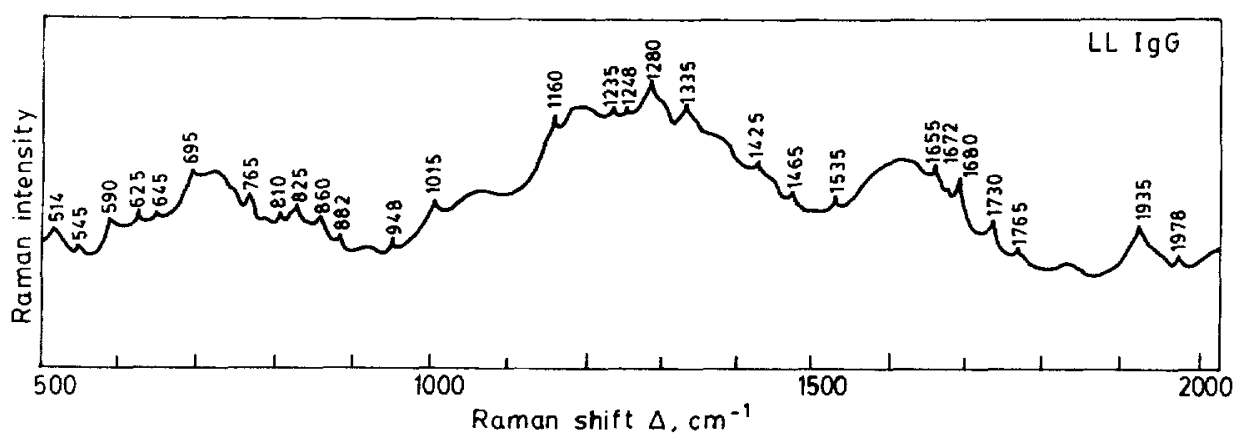

Figure 1. Laser Raman spectrum of IgG LL in $D_{2} O$, recorded from 500 to $2000 \mathrm{~cm}^{-1}$.

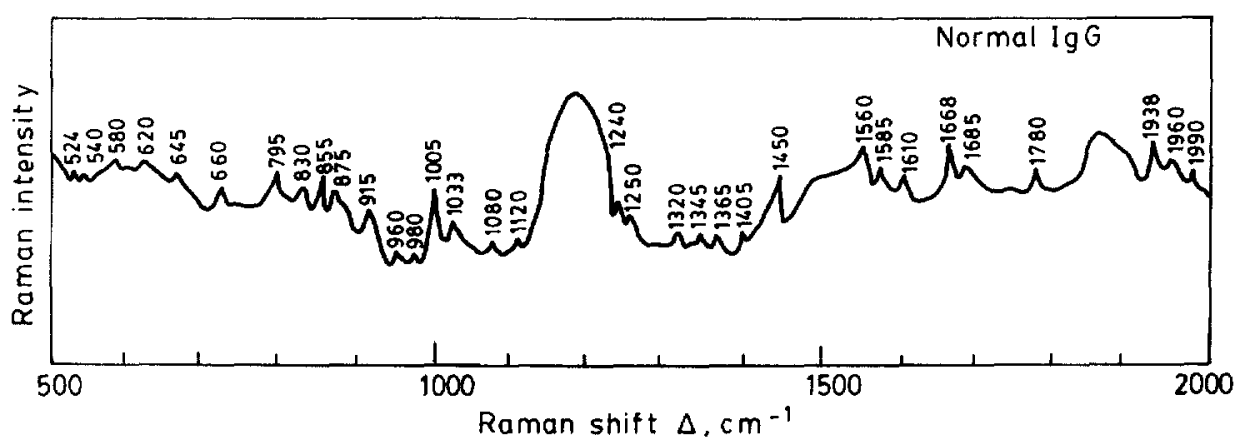

Figure 2. Laser Raman spectrum of normal human $\mathrm{IgG}$ in $\mathrm{D}_{2} \mathrm{O}$, recorded from 500 to $2000 \mathrm{~cm}^{-1}$.

$700 \mathrm{~cm}^{-1}$, whereas in the normal cases this peak is seen around $774 \mathrm{~cm}^{-1}$. Heme proteins of burn patients having 30\% burn surface area with Pseudomonas infection exhibit infrared absorption bands at 3300 (amide A), 3100 (amide B), 1650 (amide I) and $1550 \mathrm{~cm}^{-1}$ (amide II). These are the characteristic bands of the $-\mathrm{CONH}$ group.

The frequency may vary from one burn case to another due to the changes in bonding between the metal and the ligand and steric restriction about the ligand which prevent it from achieving the preferred stereochemistry. The other reasons may be weak bonding interactions, usually of the dipole-dipole type between the vibrator and its immediate environment (Clark and Hester 1986). Fermi or other vibronic interactions with neighbouring vibrators (Maxwell and Caughey 1974) may shift the frequency bands also. On the other hand, the consistency of normal hemoglobin CO stretch values at $1952 \mathrm{~cm}^{-1}$, over a wide range of species, demonstrates a remarkable consistency in binding site characterised over a broad span of evolution. Consequently, detection of the $\mathrm{CO}$ band at different frequencies in the blood represent a convenient and rapid method for the detection of abnormal hemoglobin (Aryal 1985). The CO bands appear at $1980 \mathrm{~cm}^{-1}$, in cases with $60 \%$ burns. There is clear cut distinction between the $\mathrm{CO}$ bands of the patients with different percentages of burns. Our results are in line with those reported by Gross et al (1978) stating the changes in plasma amino acids. 


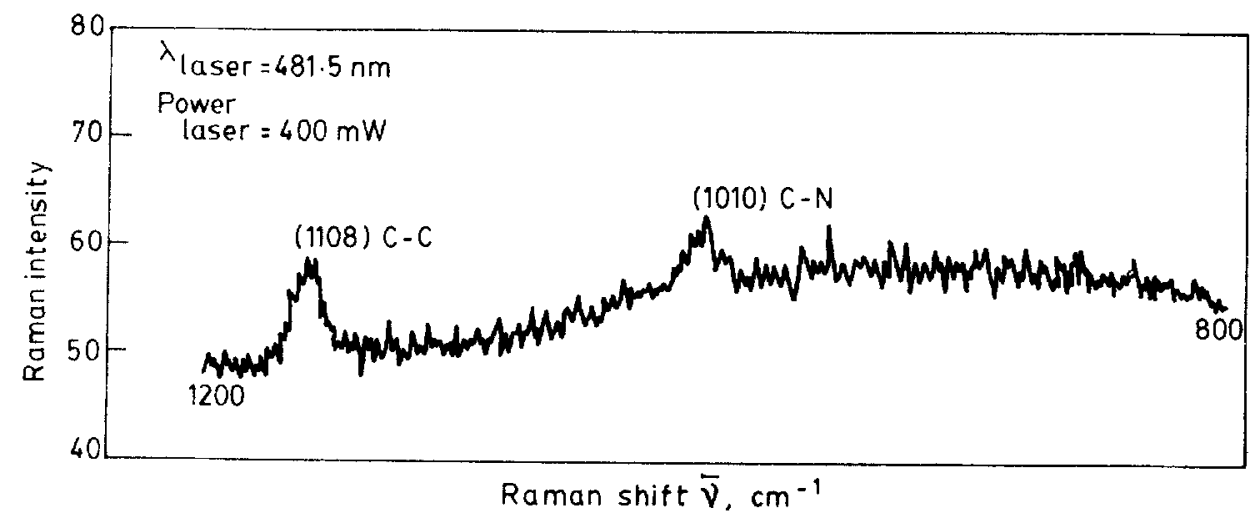

Figure 3. Laser Raman spectrum of the serum of a DMD patient in the range $800-1200 \mathrm{~cm}^{-1}$.

Raman spectra were recorded in the $\mathrm{C}-\mathrm{H}$ and $\mathrm{C}-\mathrm{C}$ stretching region $\left(800-1200 \mathrm{~cm}^{-1}\right)$ for DMD (figure 3). All samples show two prominent lines at 1010 and $1158 \mathrm{~cm}^{-1}$ due to $\mathrm{C}-\mathrm{N}$ stretch and $\mathrm{C}-\mathrm{C}$ stretch respectively. It clearly shows that conformational change marker broadens and loses intensity with denaturation.

A moderate IR signal appears at $1108 \mathrm{~cm}^{-1}$ in grand mal epilepsy (GME) due to the $\mathrm{O}-\mathrm{O}$ band, (figure 4) which is absent in normal controls (figure 5). We notice a band at $1650 \mathrm{~cm}^{-1}$, which is a typical factor needed for the identification of the alpha-helix in the LL-IgG molecule. The complexity, shape and absorption of the amide I band of secondary structure of IgG are highly heterogeneous (Kumar et al 1988). The maximum of the band occurs at $1644 \mathrm{~cm}^{-1}$ and shows an irregular conformation of IgG. FT-IR studies show that in GME amide bands appear at 3319 and $3299 \mathrm{~cm}^{-1}$ whereas no such band is observed in normal samples. Similarly the $\mathrm{S}-\mathrm{H}$ bands at 2683 and $2369 \mathrm{~cm}^{-1}$ are present in GME, whereas no such bands are seen in normal samples. Amide I and amide II bands are also found to be absent in normals but present in epileptic cases (figure 4 and figure 5). $\mathrm{O}-\mathrm{H}$ bonds are observed in normal samples, while in the majority of epileptic cases, they appear between 1803 and $1873 \mathrm{~cm}^{-1}$.

The four Raman lines appearing at $1110,1340,1410$ and $1445 \mathrm{~cm}^{-1}$ may have contributions from the $\mathrm{CH}_{2}$ group of cholesterol. The low intensities of these lines show the probable concentration of cholesterol in the erythrocytes. Previous studies (Aryal 1985) on lipid metabolism following burn injury have highlighted a fall in cholesterol levels. Cholesterol content in the erythrocytes is also reported to be depressed following injury. Shift in the positions of the peaks in the $\mathrm{C}-\mathrm{H}$ stretching region and the occurrence of a doublet may be explained in terms of disturbances of protein metabolism in burns. The enhanced protein breakdown causing protein deficiency is the possible factor affecting this region.

\section{Conclusions}

It is concluded that bands arising due to $\mathrm{O}-\mathrm{H}, \mathrm{N}-\mathrm{H}, \mathrm{C}-\mathrm{C}, \mathrm{P}-\mathrm{O}-\mathrm{C}, \mathrm{O}-\mathrm{O}, \mathrm{C}-\mathrm{H}$ bonds are severely affected in case of PTB whereas significant shifts in the strong signals are observed in the lattice water, amide I and metal ligand stretching regions 


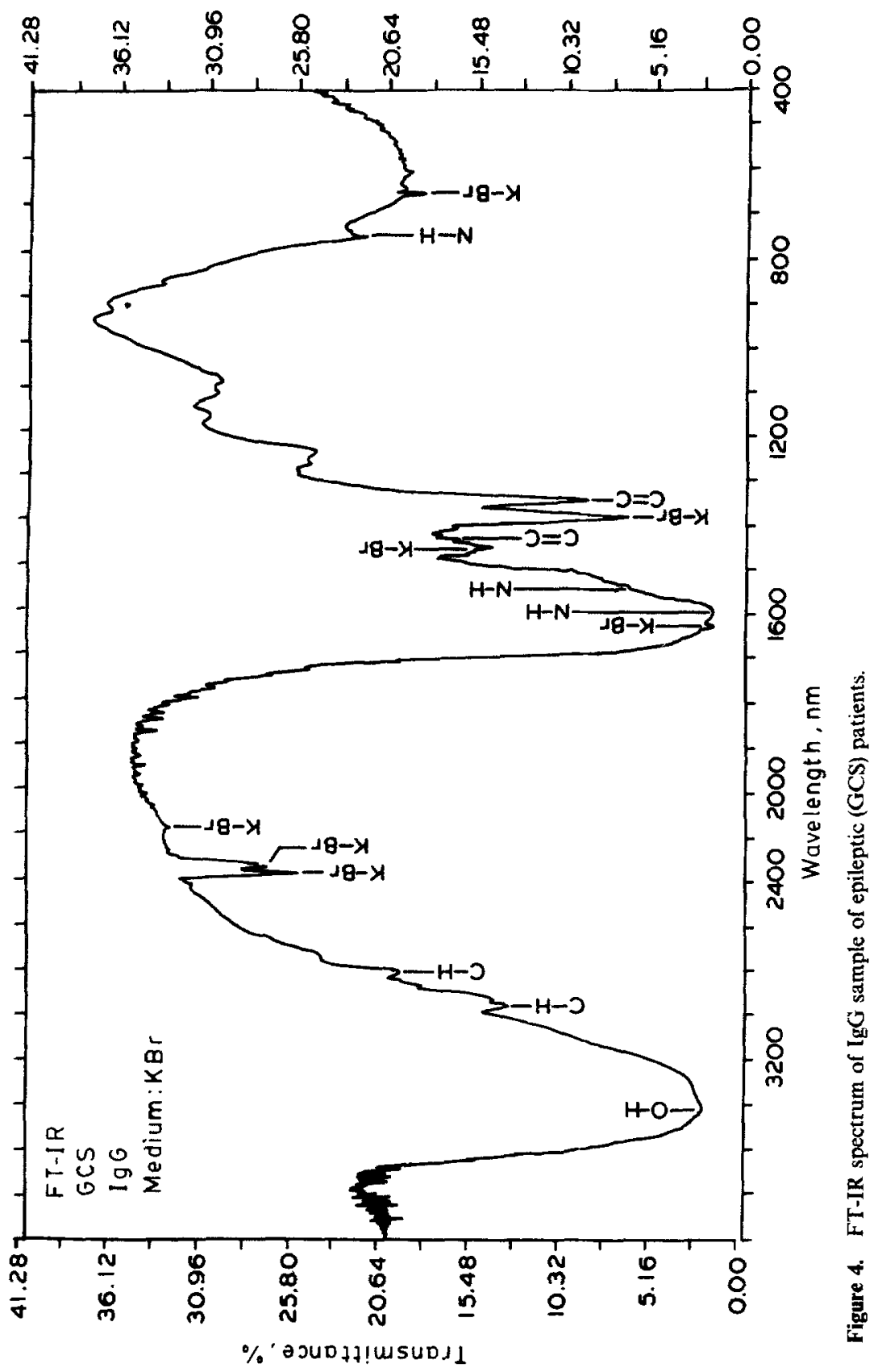




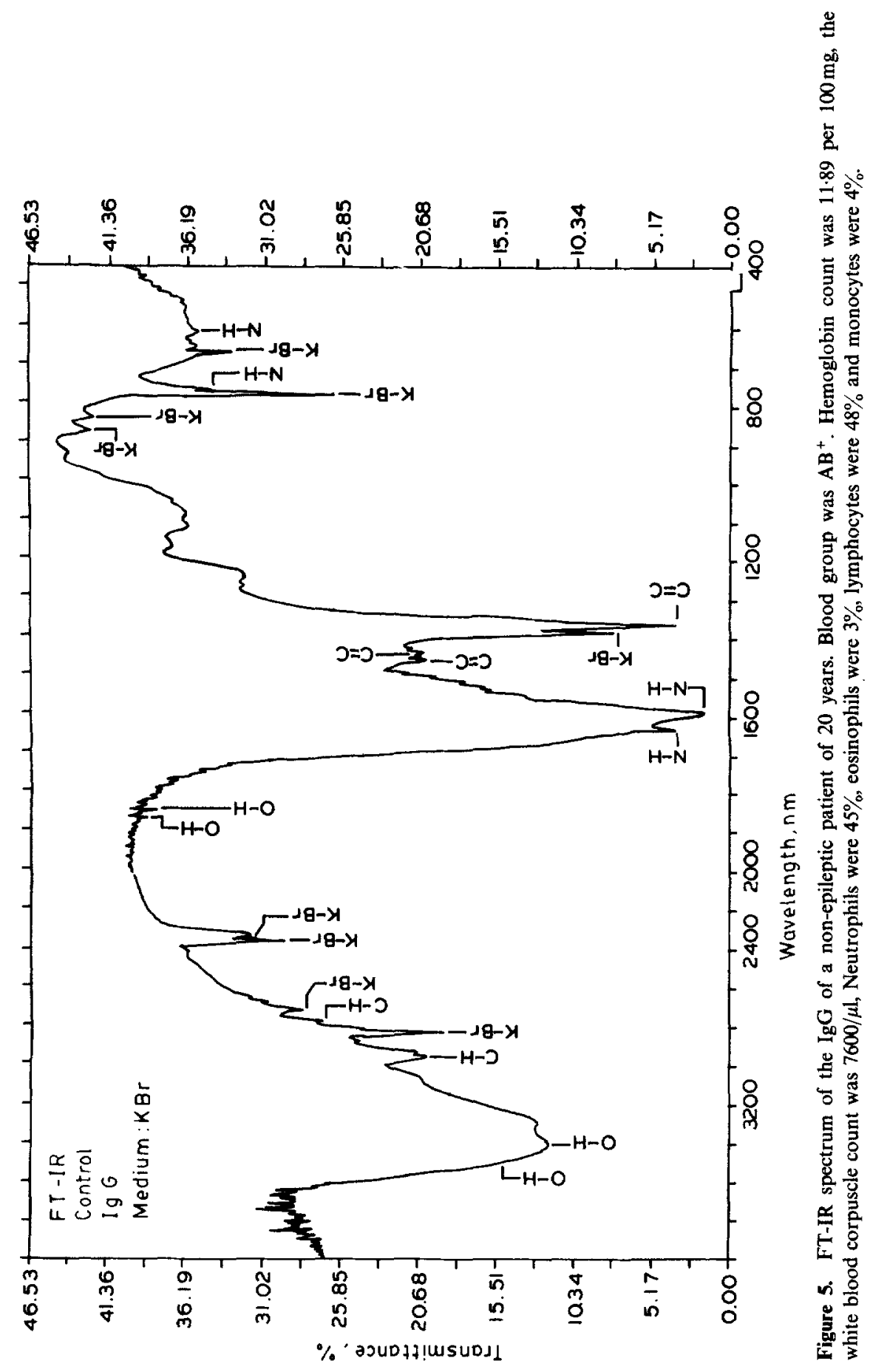


in LL cases. In burn cases, signals of amide B cyanoderivatives of myoglobin and methemoglobin amide I and amide II, carbide groups of aminoacid derivatives and peroxide compounds amide IV to amide VI groups are affected in the specific regions.

FT-IR spectra of neurological disease samples surfacially resemble those of normals, but strong peaks in the hydrocarbon region, and the disappearance of a few normal bonds are the distinct features of these samples.

Laser Raman spectra of IgG of LL samples show that the alpha-helical conformation is induced following the invasion of $M$. leprae, whereas irregular and antiparallel, beta-pleated sheet type of conformations are present in the normal samples. These conclusions are supported by our IR studies. Normal IgG exhibits ggt and tgt conformation, while LL IgG exhibits ggg and tgt conformation. The tyrosine moieties of normal IgG are exposed, while in LL they remain unexposed.

Raman spectra of erythrocyte membranes show that $\mathrm{C}-\mathrm{C}, \mathrm{C}-\mathrm{H}$, symmetric and asymmetric $\mathrm{CH}_{2}$ and $\mathrm{CH}_{3}$ vibrations are impaired following thermal injuries particularly in Pseudomonas aeruginosa infection. The lines due to $\mathrm{C}-\mathrm{H}$ and $\mathrm{C}-\mathrm{C}$ bonds may be used for the confirmation of DMD.

It is concluded that FT-IR and laser Raman spectroscopic techniques can be used as important tools for understanding the pathology of different diseases at the molecular level.

\section{Acknowledgements}

The authors would like to thank Dr R P Bhagi (Rajen Babu Tuberculosis Hospital, Delhi), Dr S P Bajaj (Department of Burns, Dr Ram Manohar Lohia Hospital, New Delhi), Dr D C Jain (Department of Neurology, Safdarjang Hospital, New Delhi) and the Medical Superintendent, Leprosy \& Tuberculosis Hospital, Tahirpur, Delhi, for providing samples and for helpful discussions. $\mathbf{S ~} \mathbf{M}$ is grateful to the Council of Scientific and Industrial Research and S K is grateful to the Indian Council of Medical Research for financial support.

\section{References}

Abaturov L V, Nozlin R S, Vengerova T L and Varshavsky T M 1969 Biochem. Biophys. Acta 154386 Amzel L M and Poljak R J 1979 Am. Rev. Biochem. 48961

Aryal S K 1985 Vibrational and magnetic resonance studies on hemoproteins and tissues afflicted with bacterial assemblies in thermal burns $\mathrm{Ph}$ D thesis, Delhi University

Bajaj M M and Khandelwal O P 1985 Physics and chemistry of human leukemia (Delhi: Academic Publications)

Clark R J H and Hester R E 1986 Advances in spectroscopy; Spectroscopy of biological systems (New York: John Wiley \& Sons) vol. 13, pp. 1-16, 347-390

Fairbanks G, Steck T L and Wallach D F H 1971 Biochemistry 102606

Freeman N K 1966 Advance Biol. Med. Phys. 4167

Grores A C, Moore J P, Woolf L I, et al 1978 Surgery 83138

Harvey A M, Johns R J, Owens A H and Ross R S 1976 The Principles and practice of Medicine (Englewood Cliffs, N J: Prentice Hall), pp. 535-609

Kumar R and Bajaj M M 1990 Vigyan 76(2) 1

Kumar S, Bajaj M M and Jain D C 1988 Current Trends in Pain Research and Therapy, Musculo-Skeletal Pain 311 
Litman GW, Litman R S, Good R A and Rosenberg A 1973 Biochemistry 12 2004-9

Maxwell J C and Caughey M S 1974 Biochem. Biophys. Res. Commun. 601309

Nikkelson R B, Verma S P and Wallach D F H 1978 Proc. Natl. Acad. Sci. 755478

Oucherlony O 1962 Diffusion-in-gel methods for immunological analysis II. (eds) P Kallos and S H Waksman VI, 30-154

Sato B K, Nishkida I, Samuls and Tyler E 1978 J. Clin. Invest. 61251

Stewart R D, Torkelson T R, Hake C L and Fercew D S 1960 J. Lab. Clin. Med. 56148

Somani $\mathrm{K} 1984$ Serum and hemoproteins in pulmonary tuberculosis using magnetic resonance, dielctric atomic absorption and immunodiffusion techniques, Ph.D. thesis, Delhi University

Verma S P and Wallach D F H 1975 Biochem. Biophys. Acta 401168

Wallach D F H, Verma S P and Fookson J F 1979 Biochem. Biophys. Acta 559153

Wier D M (ed.) 1980 Hand book of experimental immunology (Oxford: Blackwell Scientific Publications)

Wilkerson L S, Pertains R C, Roelofs R, Swift L, Dalton L R and Park J H 1978 Proc. Natl. Acad. Sci. USA 75838 